\title{
MICROFINANZAS: EVOLUCIÓN HISTÓRICA DE SUS INSTITUCIONES Y DE SU IMPACTO EN EL DESARROLLO
}

\author{
POR \\ M $^{\mathrm{a}}$ Luisa GARAYALDE NIÑO*, \\ Sara GONZÁLEZ FERNÁNDEZ ${ }^{* *}$ y \\ Juan MASCAREÑAS PÉREZ-IÑIGO***
}

\section{RESUMEN}

Este trabajo analiza desde una perspectiva histórica la evolución de las microfinanzas (con especial referencia a los microcréditos) a lo largo de su historia, centrándose especialmente en la evolución de sus instituciones y en su influencia en el desarrollo de las sociedades. En cuanto a las instituciones se analizan las cooperativas de ahorro y crédito, el crédito directo o solidario y los modelos informales (guarda monedas, banca ambulante, tontines, etc.). Se discute cómo medir su impacto en el desarrollo, cuáles han sido los aspectos positivos y negativos de las microfinanzas así como qué método es el adecuado para conseguirlo.

Palabras clave: microfinanzas, microcrédito, cooperativas de crédito, finanzas informales, tontines.

Claves Econlit: D14, G21, L31, N00, O17

\footnotetext{
* Universidad Pontificia de Comillas-ICADE. Dirección de correo electrónico: mlgarayalde@ @elefonica.net

** Universidad Complutense de Madrid. Dirección de correo electrónico: saragon@ ccee.ucm.es

*** Universidad Complutense de Madrid. Dirección de correo electrónico: jmascare@ccee.ucm.es

REVESCO No 116 - Tercer Cuatrimestre 2014 - ISSN: 1885-8031 - www.ucm.es/info/revesco

http://dx.doi.org/10.5209/rev_REVE.2014.v116.46570

Fecha de recepción: 29/08/2014

Fecha de aceptación: 08/09/2014
} 


\title{
MICROFINANCE: HISTORICAL EVOLUTION OF INSTITUTIONS AND ITS IMPACT ON DEVELOPMENT
}

\begin{abstract}
This paper examines, from a historical perspective, the evolution of microfinance (with a special reference to microcredit) throughout its history, focusing especially on the evolution of their institutions and their influence on the development of societies. As for the institutions and credit cooperatives, direct lending and informal models (coins keepers, informal banking, tontine, etc.) are analyzed. We discuss how to measure its impact on development, what are the positive and negative aspects of microfinance and what is the right method to get it.
\end{abstract}

Keywords: microfinance, microcredit, credit cooperatives, informal finance, tontine

\section{EVOLUCIÓN HISTÓRICA DEL MICROCRÉDITO}

La idea básica de lo que hoy en día se denomina "micro-crédito"1 -el concepto más conocido de las microfinanzas ${ }^{2}$ - es tan antigua como la historia escrita de la humanidad. Desde la antigua Babilonia ${ }^{3}$ pasando por las leyes religiosas hebreas, hasta desembocar en los préstamos bancarios de la Edad Media y acabando en el sistema de "tontines" o ROSCAs, muy popular en África desde hace siglos ${ }^{4}$. También se puede buscar el origen de los microcréditos en las cooperativas lecheras del Alto Jura, en Francia, en el siglo XII o en las Mutuas que se constituyen en el siglo XVI cuando la Iglesia autoriza, en Europa, los préstamos con intereses. En Irlanda, las hambrunas que se produjeron en los siglos XVII y XVIII llevaron a Jonathan Swift a tratar de romper el círculo de la pobreza a través de pequeños préstamos para lo que puso en marcha el denominado Sistema Irlandés de Fondos para Préstamos.

Es en el siglo XIX cuando se ponen las bases del microcrédito en su concepción actual $^{5}$. En dicho siglo Pierre Joseph Proudhon creó el "banco del pueblo" basado en la generalización del uso de la letra de cambio y la organización del crédito. Esta fracasada experiencia, sin embargo, mostró algo básico para el futuro de los micro-créditos: a) los

\footnotetext{
${ }^{1}$ Préstamo de pequeña cuantía concedido a personas con un acceso restringido a los créditos bancarios para que puedan financiar su propia actividad

${ }^{2}$ Otros productos son los micro-seguros, micro-ahorros y otros servicios como pueden ser las transferencias $u$ otros productos financieros destinados a los clientes con muy bajos ingresos (Servet, 2000).

${ }^{3}$ Adams, (1994) y Sery, (2012).

${ }^{4}$ Cuyas primeras referencias se hallan en la India, en los chit funds de hace 3.000 años.

${ }^{5}$ Véase Novak (1990).
} 
trabajadores tienen que tener acceso al capital para desarrollar su propia fuerza laboral sin depender de un patrón; y b) el crédito consolida los lazos sociales.

Friedrich-Wilhem Raiffeisen crea la primera cooperativa para proteger a los campesinos contra el riesgo medioambiental. Como garantía utilizaba la caución y apelaba a la conciencia de los participantes. En sus inicios esta cooperativa compraba semillas y ganado que prestaba a los campesinos a un precio muy moderado para que posteriormente ellos mismos pudiesen comprar sus propias semillas o ganado, iniciando así un círculo de desarrollo y creación de riqueza.

No es de extrañar que el ambiente imperante y las ideas de la época (nacimiento de las cooperativas y mutualidades derivadas de un movimiento filantrópico europeo, que buscaba permitir el acceso al sistema financiero a los más pobres como medio para mejorar su situación y fomentar su integración social), desembocaran en la creación en Europa de las Cajas de Ahorro $^{6}$ cuyo objetivo era ayudar a los más desfavorecidos de la sociedad.

El origen de los microcréditos se puede establecer en la creación de las Sociedades de Crédito Mutuo (1853) -debidas a los hermanos Jacob Emile y Isaac Pereire- que competían con la gran banca y canalizaban el ahorro de los rentistas tanto hacia inversiones industriales como a mejorar las condiciones de los préstamos a los pequeños empresarios combatiendo directamente la usura ${ }^{7}$, que era prácticamente la única vía de financiación que tenían los comerciantes, artesanos y pequeños industriales de la época. Es en este contexto en el que nacen las Sociedades de Socorro Mutuo que se desarrollan entre 1805 y 1847 basándose en la ayuda mutua y cooperación entre obreros y pequeños empresarios. En Francia, los hermanos Pereire tratan de organizar y racionalizar este movimiento con el objetivo de constituir estructuras bancarias destinadas a los pequeños prestatarios partiendo de los principios de las Mutualidades (creación de órganos de gestión cuyos miembros son elegidos por los participantes). Fueron estas iniciativas y otra similares, las que han permitido el desarrollo de las microfinanzas actuales. Según Sery (2012), la Grameen Bank se basa, en gran medida, en el mutualismo bancario desarrollado por los hermanos Pereire.

En el siglo XX, durante los años 1960 y 1970, las Agencias de Ayuda al Desarrollo y los gobiernos de los países en vías de desarrollo movilizaron grandes cantidades de recursos

\footnotetext{
${ }^{6}$ Véase Novak (1990)

${ }^{7}$ La información sobre los prestatarios era muy deficiente. De ahí, la necesidad de intereses desorbitados porque el riesgo de insolvencia era muy elevado.
}

REVESCO No 116 - Tercer Cuatrimestre 2014 - ISSN: 1885-8031 - www.ucm.es/info/revesco 
en programas destinados a las pequeñas empresas ${ }^{8}$. Numerosos países en vías de desarrollo crearon bancos públicos y pusieron en marcha sistemas de subvenciones de intereses, que acabarían desapareciendo ${ }^{9}$ favoreciendo con ello la creación de las instituciones de microfinanzas dirigidas a ayudar a las personas con muy pocos ingresos. Por ello, en la segunda mitad de la década de los setenta, surgen las primeras experiencias de microfinanzas en Asia y América Latina, Boyé, Hajdenberg y Poursat, (2006), con estructuras similares a las utilizadas actualmente.

El principal exponente de la experiencia asiática es el Grameen Bank ${ }^{10}$ creado en 1983 por el profesor de Economía Rural de la Universidad de Chittagong Muhammad Yunus en Bangladesh. El objetivo del banco eran los pobres -sus principales prestatarios (el 96\% mujeres)- que no tendrán que firmar un contrato formalizado para obtener su préstamo pero es necesario que cada cliente sea miembro de un grupo compuesto por cinco personas buscando, de esta forma, un compromiso moral que obligue al prestatario a someterse al control de sus pares. Desde el inicio la tasa de repago ha estado próxima al 99\% ${ }^{11}$, Yunus (1994).

La experiencia en Latinoamérica comenzó con Acción ${ }^{12}$ creada por Joseph Blatchford, una ONG cuyo objetivo es iniciar y formar a los más pobres en la ayuda mutua. En Bolivia, en 1984, se creó Prodem, ${ }^{13}$ que luego se transformará en Bancosol. Esta institución empezó a conceder préstamos de pequeña cuantía a grupos de, como mínimo, tres personas. En los cinco años posteriores a su creación, prestó por valor de 27 millones de dólares con una cuantía media de 273 dólares a más de 13.300 emprendedores (77\% mujeres), con una tasa de reembolso próxima al 100\%. En sus inicios, Prodem era una asociación sin ánimo de lucro. Pero al principio de los noventa, sus fundadores decidieron acceder al mercado de capitales para financiar sus operaciones lo que les obligó a convertirse en un banco privado de depósito y de crédito especializado en microfinanzas. Así nació Bancosol ${ }^{14}$, como banco comercial en 1992 con Prodem como principal accionista.

\footnotetext{
${ }^{8}$ Sery (2012).

${ }^{9}$ Sus resultados dejaban bastante que desear. Véase Banco Mundial, Informe sobre el Desarrollo en el mundo: sistemas financieros y desarrollo, Washington, 1989.

${ }^{10}$ Grameen significa "pueblo" "aldea" o "rural" en Bengalí.

${ }^{11}$ En palabras del propio Yunus en la cumbre de Washington de 1997 "El crédito es más importante que los negocios. Igual que la comida, el crédito es un derecho del hombre".

12 www.livingstongroupdc.com/honorable_joseph_blatchford.php

13 www.prodemffp.com

${ }^{14}$ Sus tasas de morosidad en 2011 eran muy bajas (0,77\% a treinta días). En esa fecha tenía los ratios de retorno de activos más altos del sistema bancario de Bolivia, con una calificación de Moody’s de Aaa.
} 
En África, al mismo tiempo, se crean las instituciones de microfinanzas, inspirándose en el sistema de "tontines" o ROSCAs. Estas instituciones van a animar a los beneficiarios a constituirse en grupos solidarios para garantizar el reembolso de los préstamos por parte de todos los miembros del grupo. Se puede citar como ejemplo las cooperativas Kafo Giginew que sirvieron para financiar las cosechas de algodón en Mali y Bryns en el Congo, Sery, (2012).

Al finalizar el siglo XX había alrededor de 2.000 instituciones de microfinanzas que beneficiaban a 7,6 millones de familias con sus préstamos ${ }^{15}$. En la actualidad, las mayores instituciones de microfinanzas del mundo se sitúan en Asia ${ }^{16}$; nueve de las diez mayores están en Asia oriental (tres de ellas en Bangladesh). En China, donde las microfinanzas están todavía en sus inicios, Sparreboom y Duflos (2012), las cooperativas públicas tratan de adoptar sus técnicas de gestión. En América Latina, destaca Bolivia, donde las microfinanzas tienen ya un largo historial. Acción está entre las mayores redes del mundo. En los demás países, el sector está todavía en fase de desarrollo ${ }^{17}$. En África ${ }^{18}$, las microfinanzas están implantadas en todos los países políticamente estables: en Benín, Senegal, Mali, Costa de Marfil y Kenia. En Marruecos han tenido mucho éxito. Ahí se encuentran la mitad de los micro emprendedores del mundo islámico. En Egipto y Oriente Próximo se están desarrollando rápidamente sobre todo en Jordania y Palestina.

\section{LOS PRINCIPIOS DEL MICRO-CRÉDITO}

El microcrédito está orientado fundamentalmente hacia los pobres y los excluidos a los que se les reconoce una capacidad de endeudamiento y capacidad de reembolso de los préstamos que contraigan, reconociendo su talento y sus necesidades (Collins et al, 2010) mediante unos sistemas y garantías adecuados (Boye et al, 2006). Según Yunus (2006) a través del micro-crédito, se demuestra que las personas excluidas del sistema bancario, están igual de dotadas para emprender, para gestionar sus propios negocios y para generar recursos. Más aún, se ha demostrado que tienen tasas de reembolso superiores a las de los clientes "ricos" (Yunus, 1999); además, son conscientes de que el reembolso del primer crédito

\footnotetext{
${ }^{15}$ FMI/ BM. Marco estratégico de lucha contra la pobreza: cuestiones de orden operativo, Washington, 1999.

${ }^{16}$ CGAP (Consultative Group to Assist the Poor) 2012 Annual Report. La mayor es el Grameen Bank que tiene presencia en 36.000 pueblos y presta a 6 millones de personas de las cuales el $96 \%$ son mujeres. Los préstamos medios son de 100 dólares, pudiendo ser de sólo 10 dólares. La tasa de reembolso está en el $99 \%$.

${ }^{17}$ Por ejemplo, en Méjico, los altos tipos de interés cobrados le aseguran rentabilidades muy elevadas aunque la cifra de impagados es mayor (8\%) CGAP G2P Research Project: Mexico Country Report, 2011

${ }^{18}$ CGAP (Consultative Group to Assist the Poor) 2012 Annual Report.
} 
condiciona los siguientes lo que les hace ser más solidarios y valoran especialmente su reputación porque en la reputación y en las buenas relaciones de vecindad se basan los sistemas de vida tradicionales. Esto hace que la garantía mutua, sea de un grupo de emprendedores o de un grupo de amigos, se convierta en una garantía segura aunque los garantes sean todos pobres ${ }^{19}$. Novak (1990) enumera los principios que se aplican:

- Adaptación de los préstamos a las necesidades de los clientes: cuantías pequeñas, procedimientos sencillos y plazos cortos.

- Sistema de garantías que tiene en cuenta la ausencia de bienes constitutivos de avales y la falta de recursos propios.

- El prestamista desempeña también una labor de asesor del propio negocio.

- Reembolso diseñado a la medida del cliente con pagos pequeños y frecuentes.

- Cobertura de los costes con los intereses para adquirir rápidamente autonomía operacional y financiera.

Sobre la base de estos principios se han desarrollado diferentes metodologías. Se dividen en préstamos individuales y préstamos de grupo según sean los objetivos perseguidos y el contexto en el que se concedan. Por ejemplo, los primeros se conceden, más bien, a pequeñas empresas, generalmente jurídicamente constituidas como tales, mientras que el sistema de crédito a grupos se ha utilizado sobre todo para el desarrollo de actividades generadoras de recursos (actividades tradicionales e informales), en las regiones en las que los lazos sociales son solidarios (Novak, 1990).

\section{LOS DISTINTOS TIPOS DE INSTITUCIONES DE MICROFINANZAS}

Se pueden clasificar las instituciones de microfinanzas de muchas formas siendo las más habituales las que las separan por su estatus jurídico y las que las diferencian según su actividad. Nos centraremos en este último tipo porque permite enlazarlas con su historia y sus prácticas. Desde este punto de vista se pueden clasificar las instituciones de microfinanzas en tres categorías:

\footnotetext{
${ }^{19}$ Esta es una muestra de la idea de diversificación del riesgo demostrada por (Markowitz, 1952) en la que el riesgo conjunto es menor que el riesgo medio de los individuos que conforman el grupo.
} 
1. Las mutuas y cooperativas de ahorro y crédito que utilizan el ahorro para llevar a cabo su labor de crédito.

2. Las instituciones de crédito directo que reagrupan instituciones que promueven el crédito como actividad central sin condicionarlo a la obtención previa de un ahorro.

3. Las instituciones para las que la concesión de crédito es una actividad secundaria.

Para completar esta lista habría que señalar también la existencia de las finanzas informales o autónomas ${ }^{20}$, que llevan a cabo transacciones financieras que no están reglamentadas por una autoridad monetaria central o por un mercado financiero regulado. Muy a menudo, las transacciones no están documentadas y no tienen un carácter oficial.

\subsection{Los modelos de cooperativa de ahorro y de crédito (COOPEC) y sus orígenes}

Las COOPEC son auténticas cooperativas ${ }^{21}$. Están especializadas en funciones económicas específicas, ahorro y crédito, por lo que, a menudo, tienen el estatus jurídico de instituciones financieras y están sometidas a la reglamentación bancaria nacional.

\subsubsection{Los modelos alemanes ${ }^{22}$}

Son básicamente dos: el modelo desarrollado por Raffeisen cuya primera Caja se creó en 1864 y el modelo de Schulze-Delitzch cuya primera Mutua se constituyó en 1850. A ellos hay que añadir el de Haas que se derivó del de Raffeisen y acabó fundiéndose con él.El modelo debido a Friedrich Wilhelm Raiffeisen se basa en los siguientes principios: la actividad debe estar limitada a un territorio restringido (pueblo, parroquia...), la responsabilidad de los asociados es solidaria e ilimitada, los préstamos se conceden sólo a miembros de la sociedad, no se pueden repartir beneficios entre los asociados, la gestión es gratuita en cuanto al consejo de dirección y al consejo de vigilancia. En la actualidad se utiliza en más de 700.000 cooperativas, con alrededor de 300 millones de socios en más de 100 países.

El modelo de Franz Hermann Schulze ${ }^{23}$-Delitzsch se fundamenta en los siguientes principios: garantía solidaria de los asociados y garantía del capital formado a partir de los

\footnotetext{
${ }^{20}$ Una interesante discusión sobre los posibles nombres a dar a este tipo de finanzas puede consultarse en Gentil y Fournier (1993).

${ }^{21}$ Sobre las cooperativas y las empresas de participación véase García-Gutiérrez (2002), Vargas y Lejarriaga (2002) y Lejarriaga et al. (2013)

${ }^{22}$ Artis (2006)
}

REVESCO Nº 116 - Tercer Cuatrimestre 2014 - ISSN: 1885-8031 - www.ucm.es/info/revesco 
ahorros de los mismos; estímulo al ahorro de los asociados que se traduce en una mayor participación en el capital social y que se recompensa con mayores posibilidades de obtener préstamos; y reparto de beneficios en función de la participación en el capital social ${ }^{24}$. Las ideas anteriores fueron la base de los Volksbanken (Bancos Populares) cuyo objetivo era propiciar el ahorro entre los asociados, siendo secundaria la labor de conseguir para los asociados préstamos baratos.

El tercer movimiento cooperativista alemán fue fundado por Wilhem Haas que trabajó inicialmente con Raiffeisen. Su federación admitía cualquier tipo de cooperativa agrícola, al margen de sus peculiaridades teniendo absoluta libertad a la hora de redactar sus estatutos. Un principio básico de funcionamiento de su federación era un amplio concepto de la descentralización que permitía el desarrollo de las cooperativas según sus estrategias y necesidades. Para ingresar como asociado había que suscribir una parte del capital social, pudiendo pagarse en varias entregas. En 1930 se fusionaron los sistemas Raiffeisen y Haas.

\subsubsection{El modelo canadiense: las Cajas Desjardins ${ }^{25}$}

El modelo canadiense creado por Gabriel Alphonse Desjardins nace en 1900 al constituir en Levis (Quebec). Sus objetivos son generalizar el crédito dando acceso al mismo a los obreros, pequeños agricultores y artesanos (a partir de 1914 ampliaría su actividad al sector de seguros). Los principios que sustentaban las Cajas Desjardins eran los siguientes: sólo los miembros podían hacer depósitos y solicitar préstamos; descentralización y límite territorial en torno a la parroquia o la municipalidad ya que la ayuda mutua era fundamental. Para garantizar la viabilidad de las Cajas, se exigía el pago de un derecho de adhesión en forma de participación en el capital social. En 2010 figuraba en el puesto 18 del ranking de instituciones financieras de Estados Unidos dando servicio a 5,8 millones de personas a través de 1.329 cajas populares.

\subsubsection{El traspaso de estos modelos a África ${ }^{26}$}

Estos modelos desarrollados tanto en Europa como en Canadá fueron exportados a África en dos fases; durante la colonización y tras las independencias de los distintos países.

\footnotetext{
${ }^{23}$ Schulze fue el autor de la ley de cooperativas de 1867, promulgada en Prusia, la cual fue adoptada por la Confederación Alemana del Norte en 1868.

${ }^{24}$ Schulze opinaba que incentivar las ganancias era el medio ideal para estimular la inversión de capitales de los cuales dependía la vida de la cooperativa.

${ }^{25}$ Artis (2006).

${ }^{26}$ Kengue (2012).
} 
Las cooperativas de depósito y de crédito se desarrollaron hacia 1955 en Jirapa en Ghana y posteriormente en Tanzania (1955) y en Camerún (1964). En las colonias francesas se desarrollaron dos modelos: el de un sistema bancario tradicional y el de un sistema de desarrollo más flexible. Esto llevó, según Fournier y Ouedraogo (1996), a que se pusieran sucesivamente en marcha Cajas Agrícolas $^{27}$, Sociedades Indígenas de Prevención ${ }^{28}$ y Mutualidades.

Getnet y Anullo (2012) señalan que tras esta primera generación de cooperativas, fruto del periodo colonial, se sucedieron dos modelos. La siguiente generación de cooperativas sería impulsada por los gobiernos y serían, más bien, meros instrumentos de titularidad pública para implantar los programas de planificación económica. En ese sentido, no serían auténticas cooperativas ni por su origen ni por su gestión. El impacto que tuvieron en la reducción de la pobreza también fue muy limitado lo que volvió a plantear el debate sobre las formas de organización social y económica capaces de sacar a los pobres de su situación (Francesconi y Heerink, 2010). La tercera generación sí serían auténticas cooperativas, autónomas y libres de toda influencia del Estado, que propiciaron la creación de cooperativas de Ahorro y Crédito inspiradas especialmente en las Cajas Raiffeisen (Belloncle, 1968) mientras que las cooperativas más recientes ${ }^{29}$, sobre todo a partir de los años 1988-1989 han tratado de inspirarse en las prácticas tradicionales locales de ahorro y de crédito (Servet, 1996). Así se han desarrollado las Cajas de Crédito Rural de Guinea y el proyecto de promoción del pequeño crédito rural de Burkina Faso.

\subsection{Los modelos de crédito directo o crédito solidario y sus orígenes}

Los modelos de cooperativa de ahorro y de crédito están basados en la necesidad de un ahorro, unos depósitos previos. Ese no es el caso del crédito solidario ${ }^{30}$ en el que el crédito es el elemento esencial de la actividad, siendo el depósito un aspecto complementario. Posteriormente a Bangladesh se ha desarrollado en numerosos países de Asia favoreciendo

\footnotetext{
27 Este modelo acabó fracasando porque la situación de los agricultores africanos era muy diferente a la de los europeos.

${ }^{28}$ Pero la exigencia de garantías para la concesión de los préstamos en forma de hipotecas, de garantía sobre la cosecha o el ganado, también hizo fracasar este sistema.

${ }^{29}$ Según Wanyama et al. (2008), este último tipo de cooperativas en África sí ha contribuido de forma significativa a la movilización y distribución de los recursos financieros, a la creación de empleo y a la generación de oportunidades.

${ }^{30}$ Desarrollado, en Bangladesh, por Mohamad Yunus, con el equipo de economía rural de la Universidad de Chittagong.
}

REVESCO No 116 - Tercer Cuatrimestre 2014 - ISSN: 1885-8031 - www.ucm.es/info/revesco 
especialmente a las mujeres más pobres de las zonas rurales. Actualmente, el concepto está presente, bajo distintas formas en el mundo entero.

\subsubsection{El modelo del Grameen Bank en Bangladesh ${ }^{31}$}

Desde su creación, el Grameen Bank creado por Mohammad Yunus tuvo capital mixto. En la actualidad el Estado viene a tener un $12 \%$ y los clientes el $88 \%$ restante. Cada cliente tiene una acción cuyo valor es de 100 takas. Está gestionada por un Consejo de Administración de 13 personas de los cuales 9 son accionistas y 4 están nombrados por el Gobierno. En mayo de 2010, el Grameen Bank estaba compuesto por 1.149 oficinas repartidas por 52 de los 64 distritos que configuran Bangladesh. En cuanto a los clientes (la gran mayoría mujeres), son todos pobres, campesinos sin tierras o con muy pocas, artesanos, pequeños comerciantes. Su tasa de reembolso de los créditos es del $98 \%$.

\subsubsection{El modelo de Unit Desa de la Bank Rakyat Indonesia (BRI) en Indonesia ${ }^{32}$}

La Bank Rakyat Indonesia (BRI) es un banco comercial, de titularidad del Estado, con más de 100 años de historia. En sus inicios, su objetivo era favorecer el desarrollo de las zonas rurales aunque actualmente abarca todas las áreas de la actividad bancaria y es autosuficiente. Diseñó nuevos productos tanto de ahorro como de préstamo. La tasa de morosidad se sitúa en torno al $3 \%{ }^{33}$.

\subsubsection{El modelo de la Self Employed Women Association (SEWA) en la India ${ }^{34}$}

Este banco, de carácter cooperativo, fue creado en India, en 1974, por un grupo de mujeres de la región de Ahmedabad, miembros de la Self Employed Women Association (SEWA). Los miembros son mujeres muy pobres, trabajadoras por cuenta propia, sin ningún tipo de protección social. El banco tiene por objetivo ofrecerles pequeños créditos para financiar sus actividades. Cuenta actualmente, con 11.000 miembros. El $80 \%$ de los créditos ${ }^{35}$ se conceden sin garantías pero sobre la base de referencias personales en el ámbito de la asociación. La tasa de reembolso es muy elevada, próxima al 98\%, en línea con las experiencias que se han descrito anteriormente.

\footnotetext{
31 Yunus (1994)

${ }^{32}$ Seibel (2005)

${ }^{33}$ Patten, Rosengard y Johnson (2001) demuestran que la tasa se mantuvo constante en el área de microfinanzas incluso en plena crisis económica del sud-este asiático en 1997.

${ }^{34}$ www.sewa.org

${ }^{35}$ El banco también fomenta el ahorro y ofrece seguros de asistencia social (sanidad y medicamentos)
} 


\subsection{La aplicación de los modelos en América Latina}

\subsubsection{El modelo de la Prodem - Bancosol en Bolivia ${ }^{36}$}

En su origen (1987), Prodem fue una ONG especializada en la concesión de microcréditos adaptados a las necesidades de sus clientes. En febrero de 1992, traspasó su cartera de créditos a un banco que denominó Bancosol que se ha financiado tanto con los depósitos de sus clientes como haciendo emisiones de pagarés y bonos tanto en el mercado local como en los mercados internacionales. Desde 1998, cotiza en bolsa y utiliza todos los instrumentos al alcance de una institución financiera. En 2012 gestionaba unos activos totales de 6.345 millones de pesos bolivianos con un beneficio neto de 119 millones de pesos. ${ }^{37}$

\subsubsection{El modelo de la Multi-Credit Bank en Panamá ${ }^{38}$}

Creado en 1990, el Multi-Credit Bank es un banco comercial de origen familiar. Inicialmente, se dirigía a clientes convencionales y en mayo de 1991 creó un programa autónomo denominado inicialmente "acción empresarial" y posteriormente "banco para la micro y pequeña empresa". Su objetivo era proveer de financiación a los pequeños emprendedores. En 2010, aunque los microcréditos representaban alrededor del $8 \%$ de la cartera de préstamos generaban el $21 \%$ del beneficio.

\subsubsection{El modelo de la Finansol Bank en Colombia ${ }^{39}$}

La Corporación de Acción por Bogotá (“Actuar”) creada el 7 de julio de 1988 tenía como objetivo tanto la concesión de micro-créditos como las labores de formación de los beneficiarios de los mismos. A partir de 1993 sufrió una serie de transformaciones culminando con su recapitalización en 1996 momento en que pasa a denominarse Finamerica que, además de conceder créditos a microemprendedores, también financia a pequeñas y medianas empresas.

\footnotetext{
36 www.prodemffp.com

${ }^{37}$ BancoSol, Estados Financieros 2012, www.bancosol.com.bo

${ }^{38}$ www.multibank.com.pa

${ }^{39}$ Chu (1996)
} 


\subsection{Los modelos de micro-crédito informal y sus orígenes}

Junto a los sistemas organizados de financiación a microemprendedores mostrados anteriormente existe toda una red de financiación que escapa a estas organizaciones y que se pueden denominar modelos informales ${ }^{40}$.

El primer estudio sobre este mercado se debe a Wai Utun ${ }^{41}$ que, en 1957, empezó a hablar del mercado financiero no organizado. Trataba de las prácticas usureras en Asia donde, en cada pueblo, comerciantes, prestamistas individuales, a veces profesionales, los denominados money lenders, prestaban de forma regular pequeñas cuantías de dinero, a tasas desorbitadas. El plazo era lo de menos. Se prestaba a tipos entre el 50 y el $100 \%$ tanto a un mes como a tres. En caso de retraso en el pago, se sumaban los intereses al principal que constituía la nueva deuda a pagar.

Posteriormente, Geertz (1961) analiza los aspectos financieros de las tontines o las Asociaciones Rotatorias de Ahorro y Crédito (en sus siglas en inglés ROSCAs) ${ }^{42}$. Miracle y Cohen (1980) analizaron la movilización del ahorro informal en África. Graham (1987) insiste en la amplitud del fenómeno. En un informe del Banco Mundial de 1989 se dedica un capítulo al sistema financiero informal. Germidis, Kessler y Meghir (1991) coordinan un estudio de la OCDE en la misma línea. Ghate (1992) analiza los mercados financieros informales en los países presentes en el seminario organizado por el Asian Development Bank en Manila. Según Vonderlack y Schreiner (2003), los costes de transacción son bajos lo que supone una clara ventaja competitiva. Pero también presentan inconvenientes: no preservan el anonimato de las partes y existe mucha presión social porque todos se conocen entre sí. A pesar de ello, todavía hay un alto grado de inseguridad tanto en la garantía de los depósitos en el caso de ahorro como de la localización de los prestatarios.

Gentil y Fournier (1993) clasifican estas prácticas en cinco categorías: los guarda moneda, los usureros, las tontines, los banqueros ambulantes y las cajas de solidaridad. Adams (1994) los clasifica según sean organismos sofisticados pero no reglamentados, prestamistas profesionales, comerciantes, aquellos que conceden créditos con prenda, agentes

\footnotetext{
${ }^{40}$ Esta financiación informal forma parte de un concepto más amplio conocido como shadow banking o banca en la sombra; véase Parramón (2014)

${ }^{41}$ Utun (1957).

${ }^{42}$ Estas prácticas adquirieron notoriedad a partir de 1977, cuando Fináfrica lanzó en Milán la revista especializada Savings and Development que trató reiteradamente este tema.
} 
especializados en préstamos, propietarios, familiares y amigos, guarda monedas, grupos de ahorro o asociaciones rotatorias de ahorro y crédito. Holst (2003), por su parte, clasifica las distintas prácticas de finanzas informales según el tipo de relación que existe entre los distintos participantes en la operación es decir entre el prestamista, el prestatario y el ahorrador. En cuanto a Montel, Agenor, y Haque (1993) recogen cuatro categorías: los préstamos ocasionales (parientes, amigos, vecinos...), los préstamos vinculados a una actividad (comerciantes, propietarios de la tierra, empresario a su empleado...), los préstamos grupales o asociativos (tontines, asociaciones de crédito y ahorro), los préstamos concedidos por profesionales (banqueros ambulantes, usureros...).

Hugon (1996) distingue tres tipos de prácticas: las instituciones comunitarias que se basan en relaciones de parentesco o clan sin adhesión voluntaria que se pueden interpretar como apoyos intergeneracionales; las asociaciones de crédito rotativo que requieren una decisión individual voluntaria pero que tienen un carácter temporal; los prestamistas no oficiales que se basan en conceptos financieros puros pero que se encuentran al margen de las instituciones reguladas. Servet (1995) separa las formas individuales de las colectivas. En las individuales se encuentran los familiares, las autoridades políticas o religiosas locales (sacerdote, imán, jefe tribal), los amigos, los banqueros ambulantes y los guarda monedas. Las formas colectivas serían las tontines y demás formas asociativas. Según Lelart (2000) no se trata tanto de actividades de préstamo o ahorro como de aspectos de la vida social. Así entendería los préstamos entre familiares o los concedidos por los comerciantes a sus buenos clientes. Otras, sin embargo, no son directas. Requieren de un intermediario (banquero ambulante...) que pone en relación a las personas con ahorros de las que necesitan recursos. Estas sí serían propiamente financieras. En esta misma línea se encuentran los trabajos de Lelart (1993 y 1995) y Bekolo Ebe (1989).

\subsubsection{Los préstamos privados: préstamos de familiares y préstamos profesionales}

Los préstamos personales suelen carecen de intereses asociados y, a veces, ni tan siquiera hay obligación de devolución del principal. La contraprestación es social pero crea una cierta obligación moral en el beneficiario que, a su vez, tendrá que acudir en ayuda de cualquier otro familiar necesitado. Los préstamos profesionales son los que se generan a raíz de una determinada actividad económica y, aquí también, hay una relación personal entre el prestamista y el beneficiario. En esta categoría, se englobarían los préstamos entre comerciantes y cliente, entre propietario de la tierra y agricultor, entre proveedores de materia 
prima y artesanos. Estos préstamos suelen ser de muy corto plazo y pueden devengar intereses o estar implícitos en el precio de los bienes adquiridos.

\subsubsection{Los bancos informales: los guarda moneda y los banqueros ambulantes}

Gertrud (1994) define los bancos informales como organismos en los que los participantes aceptan depositar regularmente sus ahorros sobre una base contractual. El ahorro puede utilizarse para conceder préstamos a los miembros del organismo o a terceros, sea con intereses o sin ellos. Se incluye en esta categoría a los guarda monedas y a los banqueros ambulantes. Los guarda monedas son personas a las que otras personas confían sus ahorros. Se puede tratar de una autoridad familiar (padre, madre, tío...), de una autoridad religiosa a política (sacerdote, imán, jefe tribal...) o de un amigo que tenga una situación económica estable. El punto en común de todas estas personas es que gozan de una buena reputación y que hay una relación personal de confianza entre el depositante y ellas. No está documentado el uso que dan los depositarios a estos fondos, parece que los conservarían tal cual y los devolverían sin añadirles intereses a una fecha convenida o a petición del depositante. Los banqueros ambulantes son figuras con una larga tradición histórica en África ${ }^{43}$ y hacen de auténticos intermediarios entre los ahorradores y los demandantes de financiación cobrando por su actividad.

\subsubsection{Las tontines o $\mathrm{ROSCAS} \mathrm{S}^{44}$}

Se trata de una práctica muy extendida en muchos países europeos, de América Latina y sobre todo de Asia (en Japón se denominan Kou, en China Hui, en Corea Kye, etc). Según Mayoukou (1994), las tontines de trabajo ya existían en África ${ }^{45}$ antes de la introducción de la moneda, momento a partir del cual se han transformado en tontines de dinero. Son las prácticas de finanzas informales más utilizadas. Según Hugon (1996), se pueden definir las tontines como un fondo de ahorro rotativo, cuyas aportaciones se entregan a cada uno de los miembros, según un orden preestablecido aunque revisable. Todos van a tener, por lo tanto, un carácter tanto de depositantes como de prestatarios. No se pagan intereses por los préstamos ni se remunera el ahorro. Se organizan habitualmente por un grupo homogéneo de personas, reunidas por sus lazos familiares o de proximidad religiosa, étnica, política, social o

\footnotetext{
${ }^{43}$ Su origen se sitúa en Nigeria, en la etnia Nago.

${ }^{44}$ El origen de la palabra tontine se sitúa en una práctica asociativa de ahorro localizada en Nápoles y desarrollada por el banquero italiano Lorenzo Tonti en 1653. Véase Hugon (1996).

${ }^{45}$ En África está generalizada bajo diversos nombres: esussu, cha, adashi, osussu, susu, essu, esso, asossou, sedjo, djonou, edjo, adokavi, etc.
} 
de amistad. La cohesión social del grupo es un factor clave de este sistema ya que se basa en la confianza y en la palabra dada. El control social es muy fuerte y la disciplina financiera muy estricta. Se pueden imponer sanciones en caso de impago. El sistema es rápido, simple y fiable siendo la tasa de reembolso casi del $100 \%$. Se pueden distinguir tres tipos de tontines:

- Tontine mutua o giratoria. Según Lelart (1990 y 2000) se trata de una asociación de personas que se conocen bien, deseosas de prestarse y de recibir préstamos de poco monto durante un cierto tiempo. Cuando cada uno ha recibido tanto como ha depositado, el grupo se deshace, a menos que los participantes quieran hacer una segunda ronda.

- Tontine comercial. Esta figura es parecida a la del banquero ambulante. El tontinero pasa a recoger con una periodicidad regular ya sea diaria o semanal una cantidad de dinero preestablecida. Al cabo de un tiempo también prefijado, normalmente un mes, el banquero devuelve la cantidad acumulada restando una comisión por su custodia. Para fidelizar a sus clientes, a menudo, el tontinero les concede préstamos (Lélart, 1996).

- Tontine financiera. Lo que caracteriza a la tontina financiera y la diferencia de la anterior es el cobro de intereses en los préstamos y la remuneración de las cantidades depositadas. A menudo, se subasta entre los miembros el destino de los fondos. Todos los clientes del tontinero se conocen (Lélart, 1991 y 1996).

\section{EL IMPACTO DE LAS MICROFINANZAS EN EL DESARROLLO}

\section{1. ¿Cómo medir el impacto?}

Varios estudios muestran el impacto de las microfinanzas en el desarrollo y la mejora de condiciones de vida de sus usuarios (Dupas y Robinson, 2008; Udry y Aryeetey, 2010, Banerjee et al, 2009). Sin embargo, una duda que se plantea es con qué finalidad se hace esta medida del impacto. La mayoría de la investigación se ha centrado en cómo mejorar el sector de las microfinanzas en sí más que en cómo mejorar el impacto que produce en sus usuarios (Hulme, 1997); se trata no sólo de medir los aspectos financieros sino también los producidos en la vida de las personas así como en su barrio o región (Van Rooyen, Stewart y De Wet, 2012). Hay varios autores que se han preocupado de medir el impacto real de los programas de microfinanzas sobre sus usuarios: Hulme (1997), Mayoux (1997), Cohen (1998), Himes y Servon (1998), Cheston, Reed y Harper (1999), y Van Rooyen, Stewart, De Wet (2012). 
La publicación de tres evaluaciones de impacto de los programas de micro-créditos en India, Filipinas y Marruecos han puesto en entredicho el valor del microcrédito para los pobres (Karlan y Zinman, 2009; Banerjee et al., 2009) y, por último, Bennett (2009) que ha sido más radical declarando que las microfinanzas son un fracaso. Un grupo de investigadores $^{46}$ del CGAP opina que las instituciones de microfinanzas han centrado sus recursos en el seguimiento ${ }^{47}$ más que en las evaluaciones externas. Para ello proponen que las evaluaciones de impacto utilicen estudios aleatorios controlados, RCT (random controlled trial), una metodología de evaluación que asigna de forma aleatoria una intervención a un grupo de tratamiento y priva de ella a un grupo de control (de forma similar a cómo se realizan los estudios en los ensayos clínicos y farmacológicos). Se pueden clasificar los métodos de medición de impacto en tres grandes categorías:

1. Métodos cualitativos: se centran en los procesos, los comportamientos y las condiciones tal y como los perciben los entrevistados (Adjei et al, 2009).

2. Métodos cuantitativos no experimentales: ofrecen pruebas de cambios en las vidas de los clientes. Puede haber un sesgo en la selección de la muestra (sesgo de selección), la ubicación, etc., (Chen y Snodgrass, 2001; Khandker, 1998; Bruhn y Love (2009) y Townsend y Kaboski, 2009).

3. Métodos cuantitativos experimentales (estudios aleatorios controlados RCT): demuestran la causalidad de una intervención en las vidas de los clientes en comparación con un grupo de control (Karlan y Zinman, 2010; Bauchet et al, 2011 y Banerjee et al, 2009).

Los estudios que realizan pruebas experimentales con diferentes periodos de pago, el uso de mecanismos de compromiso para el ahorro o la elasticidad de los tipos de interés constituyen algunos ejemplos de cómo se utilizan los estudios aleatorios controlados para mostrar el modo en que pueden mejorarse los servicios (Gine y Karlan, 2006; Ashraf, Karlan y Yin, 2006). Los estudios aleatorios controlados también plantean cuestiones importantes, a

\footnotetext{
${ }^{46}$ Bauchet, Marschall, Starit, Thomas y Yalouris

${ }^{47}$ Inicialmente ese seguimiento se limitaba a indicadores financieros y posteriormente se incorporaron observaciones de los cambios registrados en una serie de dimensiones sociales identificadas y acordadas en el marco del grupo de acción sobre el desempeño social. Actualmente, un grupo compuesto por más de 350 IMF está presentando al Microfinance Information Exchange (MIX) un conjunto de indicadores sociales, junto a los financieros.
} 
menudo, con una vertiente ética en la medida en que hay que decidir quién recibe el servicio y quién no y por lo tanto sirve de grupo de control.

\subsection{Impactos detectados}

Las microfinanzas funcionan de forma diferente en las distintas regiones donde la densidad de población, la actitud frente a las deudas, la cohesión de los grupos, el desarrollo empresarial y los proveedores de servicios financieros son muy dispares (Armendariz de Aghion y Morduch, 2005; Fischer y Ghatak, 2011; Microfinance Information Exchange y CGAP, 2011). Duvendack et al (2011) señalan que la mayoría de los estudios de impacto realizados en Asia han obtenido resultados contradictorios.

Las microfinanzas destinadas a los pobres $^{48}$ han permitido aliviar adecuadamente su situación mejorando su nivel de vida (CGAP, 2003; Robinson, 2001; Yunus, 1999). Las microfinanzas también han tenido otras consecuencias financieras como el incremento del ahorro o la acumulación de activos como pequeña maquinaria o muebles e impactos no financieros, en la salud, la seguridad alimenticia, la nutrición, la educación, la creación de empleo, la situación de la mujer y la cohesión social (Afrane, 2002; Barnes, 1996; Barnes y Keogh, 1999; Beck, Demirgue-Kunt y Levine, 2004; Hietalahti y Linden 2006; Hossain y Knight, 2008; Khandker, 2001; Odell, 2010; Schuler, Hashemi y Riley, 1997, Unicef, 1997; Wright, 2000). La idea que subyace en todos estos estudios es que proveyendo de servicios financieros a los pobres, estos administran su dinero de forma diferente, invirtiendo, comprando activos productivos, mejorando su rendimiento personal, su auto estima, etc.

Pero otros estudios no dan una versión tan claramente positiva de las microfinanzas. Algunos indican impactos positivos para los pobres pero no para los más pobres (Copestake, Bhalotra y Johnson, 2001; Hulme y Mosley, 1996; Morduch, 1998; Mosley y Hulme, 1998; Zaman, 2001). Otro señala que han mejorado los ingresos pero no lo suficiente (Rutherford 1996). La situación de la mujer también parece haber mejorado pero aquí también perduran todavía fuertes desigualdades (Husain, Mukherjee y Dutta, 2014; Mayoux 1999; Rahman 1998). El dinero dedicado a las microfinanzas podría ser más efectivo con otro tipo de intervenciones (Karnani, 2007) sobre todo si se combina con programas de salud o educación (Lipton, 1996).

\footnotetext{
${ }^{48}$ Es decir a aquellas personas que viven con menos de dos dólares al día, especialmente cuando se trata de micro-créditos de entre 50 y 1.000 dólares.
} 
Por último, para otros autores las microfinanzas son claramente negativas. Fomentan la explotación de la mujer, incrementa las desigualdades de ingresos, crea relaciones de dependencia y dificulta el desarrollo sostenible (Adams y Von Pischke, 1992; Bateman y Chang, 2009; Copestake, 2002). Las microfinanzas no solo están puestas en tela de juicio por su falta de efectividad en la disminución de los niveles de pobreza sino que también se cuestionan desde un punto de vista ideológico (Bateman, 2010; Dichter, 2007; Fernando, 2006; Roy, 2010). La reciente crisis ha impactado duramente en miles de personas sobre endeudadas a través de mecanismos de microcréditos con sus consiguientes implicaciones para su bienestar y el de su comunidad (Bahía, 2011). También se cuestiona si éticamente es aceptable ganar dinero prestando a los pobres y si el desarrollo del sector ha hecho perder de vista los objetivos originales (Fernando, 2006; Karnani, 2009; Weber, 2006; Senigaglia, 2008). Especialmente en India, se ha pedido que se regule el sector teniendo en cuenta el número de negocios que han quebrado y el incremento de suicidios entre los clientes de microcréditos.

El primer estudio basado en RCT (Randomized Controlled Trials) en India y Filipinas (Banerjee et al., 2009; Karlan y Zinman, 2010) fracasó en su intento de encontrar pruebas de que las microfinanzas disminuían los niveles de pobreza. Obtuvieron una respuesta claramente defensiva por parte de la industria (Acción Internacional et al., 2010). Los estudios cuyo objeto ha sido África también son contradictorios. Algunos demuestran que las microfinanzas disminuyen la pobreza y mejoran la calidad de vida de los clientes y otros no (Ashraf, Gine y Karlan, 2008; Barnes, Keogh y Nemarundwe, 2001; Dupas y Robinson, 2008; Gubert y Roubaud, 2005; Nanor, 2008). Dos estudios demuestran que los granjeros que utilizan microcréditos son capaces de diversificar sus cosechas y por lo tanto no dependen tanto de un solo producto (Barnes, Gaile, y Kibombo, 2001; Barnes, Keogh, et al., 2001) pero solo el segundo de estos trabajos señala que esta diversificación aumenta los ingresos de dichos granjeros. Otro estudio sugiere que los clientes de microfinanzas gestionan mejor sus negocios que los del grupo de control pero los datos no son estadísticamente significativos (Gubert y Roubaud, 2005). Otro trabajo señala que cuanto más tiempo un cliente utilice las microfinanzas peores resultados obtiene en su actividad (Nanor, 2008). Para otros investigadores, el impacto de las microfinanzas en los resultados del negocio es neutral (Dupas y Robinson, 2008). En lo que sí parecen coincidir todos los estudios es en que el uso de las microfinanzas aumenta el nivel de ahorro de las familias (Adjei et al, 2009; Barnes, Gaile et al. 2001; Dupas y Robinson, 2008; Ssewamala et al., 2010). También demuestran que las microfinan- 
zas tienen como consecuencia un incremento de los activos en propiedad en el corto plazo pero que esa tendencia a la acumulación no se mantiene en el tiempo (Adjei et al., 2009; Brannen, 2010).

Por otra parte, hay evidencias que demuestran que los microcréditos suponen una mejoría en los cuidados médicos y en la salud de las familias de los clientes (Lacalle Calderon, Rico Garrido y Duran Navarro, 2008) así como un mayor gasto sanitario (Adjei et al, 2009; Brannen, 2010; Dupas y Robinson, 2008). La duración de los programas de microfinanzas no inciden en este impacto (Adjei et al, 2009). Las microfinanzas mejoran la salud de los niños gracias al mayor uso de medidas preventivas como la utilización de mosquiteras (Brannen, 2010) así como por la mejoría en la nutrición (Doocy et al, 2005). En este último punto, Doocy y sus compañeros de investigación señalan que existen grandes diferencias según las zonas geográficas analizadas. Afirman, además, que los niños están mejor alimentados cuando son las madres las clientes de los micro-créditos (Doocy et al, 2005). Los micro-créditos también impactan en la salud sexual y en la incidencia del sida (Pronyk et al, 2008; Ssewamala et al, 2010).

En cuanto a la alimentación, los estudios demuestran que mejora su calidad pero no su cantidad (Brannen, 2010; Doocy et al, 2005, Lacalle Calderon et al, 2008). En Zimbabwe los miembros de un programa de microfinanzas cambiaron sus hábitos alimenticios incorporando mayor cantidad de carne, pescado, pollo y leche en relación a los que no participaron en dicho programa o los que lo abandonaron (Barnes, Keogh et al, 2001). Este estudio, sin embargo, contrasta con los datos recogidos en Etiopía (Doocy et al, 2005) y Ghana (Nanor, 2008) que muestran escasas diferencias en la dieta de los usuarios de microfinanzas y la muestra de contraste.

El impacto de los micro-créditos en la educación es contradictorio. Algunos estudios demuestran que la inversión en educación de los niños es mayor en los clientes de microfinanzas (Adjei et al, 2009; Lacalle Caderon et al, 2008) pero otros no constatan esa consecuencia (Brannen, 2010; Gubert y Roubaud, 2005). Datos de un estudio llevado a cabo en Malawi señalan que los hijos de los usuarios de micro-créditos faltan mucho más a la escuela. Los chicos repiten curso con más frecuencia y el porcentaje de niñas escolarizadas es menor (Shimamura y Lastarria-Cornhiel, 2009). El porcentaje de abandono de la escuela de niñas entre 6 y 16 años es mayor, según otro estudio, en las hijas de clientes continuados de micro-créditos que en aquellos que nunca han utilizado esta fuente de financiación o no 
recurren a ella de forma reiterada (Barnes, Keogh et al, 2001). Este mismo estudio refleja que el impacto en niños y niñas es diferente. Para los chicos la incidencia es positiva mientras que para las niñas es negativa o neutral. En Uganda, un estudio sobre el microahorro pone de manifiesto que los que lo utilizan tienen más posibilidades de seguir con la escolarización en el segundo ciclo de la enseñanza que el grupo de control (Ssewamala et al, 2010). En conclusión, el impacto de la microfinanzas en el ámbito de la educación es variado, con limitadas experiencias positivas y con claros indicios de que puede ser perjudicial para los hijos de los usuarios. Como el uso de los microcréditos no fomenta el trabajo infantil (Shimamura y Lastarria-Cornhiel, 2009) no queda muy claro por qué se saca a los niños, en particular a las niñas, del colegio.

De las investigaciones llevadas a cabo en la zona de África sub-sahariana, no hay datos que certifiquen que las microfinanzas tienen un impacto positivo en las condiciones de vida de las mujeres. En Uganda, un estudio señala que la capacidad de toma de decisión de la mujer está mejorando pero es difícil saber si se debe solo al uso de los microcréditos (Yaron, 1994). En la encuesta llevada a cabo en Sud-África se ve un descenso de la violencia de género pero tampoco se puede establecer una relación directa con el uso de microcréditos (Pronyk et al, 2008).

En cuanto al acceso a la vivienda, los estudios señalan que sí se produce un impacto positivo. Los participantes en un programa de microfinanzas en Zanzibar han podido invertir en un porcentaje superior al grupo de control y adquirir su propia casa (Brannen, 2010). En Ruanda, las reformas y mejoras de las viviendas fueron superiores en los usuarios de microfinanzas que en los no clientes (Lacalle Calderon et al, 2008). Barnes, Gaile et al (2001) llegan a conclusiones en la misma línea.

\section{CONCLUSIONES}

A la vista de lo mostrado un modelo de microfinanzas adecuado debe contar con la confianza de sus usuarios, proporcionar impactos positivos en la vida de los beneficiarios y justificar el uso de las donaciones en cuanto a la ayuda lograda por las inversiones que han generado (Krahnen y Schmidt, 1994). 
Para medir adecuadamente el impacto se deben utilizar indicadores de rentabilidad inspirados en ratios financieros como es el caso del SROI (social return on investment) ${ }^{49}$. Yaron (1992) desarrolló un Índice (SDI) para medir la dependencia de un programa de las ayudas o subvenciones en la cobertura de sus gastos operativos y financieros incluyendo un coste implícito de los recursos propios. Se utiliza entonces este índice para establecer el tipo de interés que se cargará a los préstamos independizando por lo tanto a la institución de los subsidios. Está diseñado para que se pueda calcular en todo tipo de programas ya sean llevados a cabo por grandes instituciones o por organizaciones pequeñas. Se calcula sobre la base de datos históricos ajustados por inflación y coste de los recursos.

Numerosos autores (Hulme y Mosley, 1996; Khandker, 1998; Yaron 1992 y 1994) insisten en la necesidad de cargar un tipo de interés que cubra todos los costes operativos y financieros para garantizar la sostenibilidad. El CGAP también incluye este requisito entre sus recomendaciones. Sin embargo, Schreiner (1997) advierte que siguiendo este procedimiento se puede tender a perder eficiencia en las operaciones. También se puede dejar de dar servicios a aquellos grupos de posibles beneficiarios que residen en zonas rurales con baja densidad de población, deficientes infraestructuras o muy bajo poder adquisitivo y por lo tanto solicitantes de préstamos de muy bajo importe que van a generar unos costes de gestión en la concesión de los créditos muy elevados. A menudo son precisamente los más necesitados, los que se encuentran en estos entornos.

En conclusión, el modelo se considerará adecuado y sostenible en la medida en que tenga un impacto positivo en el nivel de vida de los beneficiarios y en cuanto genere un efecto multiplicador de las donaciones y por lo tanto un uso eficiente de los recursos. Se considera que este efecto multiplicador es un argumento diferencial que puede atraer a los donantes. De esta forma se cumple con la definición de sostenibilidad (Woller et al. 1998): habilidad del programa para producir outputs que estén suficientemente valorados por los beneficiarios y por los suministradores de capital como para que el programa siga recibiendo recursos e inputs que le permitan proseguir con su desempeño.

\footnotetext{
${ }^{49}$ Narrillos (2010) y Narrillos (2013). Sobre la Economía Social y los modelos sostenibles puede consultarse Chaves y Monzón (2012), Chaves et al (2013) y Fecher et al (2012)
} 


\section{BIBLIOGRAFÍA}

ACCION et al (2010) Measuring the impact of microfinance: our perspective, Abril

ADAMS D. (1994) Une nouvelle vision de la finance informelle, Finance informelle dans les pays en développement, pp. 20-26.

ADAMS D. y VON PISCHKE J. (1992) Micro-enterprise credit programs: dejá vu, World Development, pp. 1463-1470.

ADJEI, J.K. et al, (2009) The role of microfinance in asset building and poverty reduction: the case of Sinapi Aba Trust of Ghana, Brooks World Poverty Institute, Manchester.

AFRANE S. (2002) Impact assessment of microfinance interventions in Ghana and South Africa: a sintesis of major impacts and lessons, Journal of Microfinance, pp. 37-58.

ARMENDARIZ de AGHION B y MORDUCH J. (2005) The economics of microfinance, The MIT Press, Cambridge, MA.

ARTIS A. (2006) La finance solidaire: un systéme de relations de financement, Université de Grenoble.

ASHRAF N., GINE X y KARLAN D. (2008) Finding missing markets (and a disturbing epilogue): evidence from an export crop adoption and marketing intervention in Kenya, World Bank, Washington DC.

ASHRAF N., KARLAN D. y YIN W. (2006) Tying Odysseus to the mast: evidence from a commitment savings product in the Philippines, Quarterly Journal odf Economics. 121 (2): 635-672.

BAHIA, B (2011) El Microcrédito como instrumento al servicio de las entidades de Economía Social. Especial referencia al instrumento europeo de microfinanciación Progress. Revesco. Revista de Estudios Cooperativos n 106, pp. 33-54.

BANERJEE A. V., et al. (2009) The miracle of microfinance? Evidence from randomized evaluations, CGAP, Washington DC.

BARNES C. (1996) Assets and the impact of micro-enterprise finance programs, AIMS paper, Washington DC.

BARNES C., GAILE G. y KIBOMBO R. (2001) The impact of three microfinance programs in Uganda, Development Experience Clearinghouse, USAID, Washington DC.

BARNES C. y KEOGH E. (1999) An assessment of the impact of Zambuko's microenterprise programs in Zimbabwe: baseline findings, Washington DC.

BARNES C., KEOGH E. y NEMARUNDWE N. (2001) Microfinance program clients and impacts: an assessment of Zambuko Trust Zimbabwe, AIMS, Washington DC. 
BATEMAN M. (2010) Why doesn't microfinance work? The destructive rise of local neoliberalism, Zed Books, New York.

BATEMAN M. y CHANG H. (2009) The microfinance illusion. Disponible en: www.econ.cam.ac.uk/faculty/chang/pubs/microfinance.pdf .

BAUCHET J., et al. (2011) Latest findings from randomized evaluations of microfinance, CGAP Publications.

BECK T., DEMIRGUE-KUNT A. y LEVINE R. (2004) Finance, inequality and poverty: cross country evidence, World Bank policy research working paper 3338, Washington DC World Bank.

BEKOLO EBE (1989) Le système des tontines: liquidité, intermédiation et comportement d'épargne, Revue d’Economie politique, $\mathrm{N}^{\circ} 4$, pp. 616-639.

BELLONCLE G. (1968) Le crédit agricole dans les pays d"expression française au sud du Sahara, Rome, FAO.

BENNETT D. (2009) Small change, billions of dollars and a Nobel Prize later, it looks like microlending doesn't actually do much to fight poverty, Boston Globe, 20 septiembre.

BOYÉ S., HAJDENBERG J., POURSAT C. (2006) Le guide de la Microfinance, Editions D’Organisation, Paris.

BRANNEN C. (2010) An impact study of the Village Savings and Loan Association (VSLA) program in Zanzibar, Tanzania, Wesleyan University.

BRUHN M. y LOVE I. (2009) The economic impact of banking the unbanked: evidence from Mexico, documento de trabajo sobre investigaciones relativas a políticas de desarrollo $\mathrm{n}^{\mathrm{a}} 4981$ WPS, Washingngton, DC, Banco Mundial.

CGAP, Microfinance consensus guidelines, World Bank, Washington DC, 2003.

CHAVES ÁVILA, R; MONZÓN CAMPOS, J. L (2012) Beyond the crisis: the social economy, prop of a new model of sustainable economic development. Service Business. An international journal, (vol 6:1). Disponible en: http://www.springerlink.com/content/1862-8516/6/1/

CHAVES ÁVILA, R; MONZÓN CAMPOS, J. L; PÉREZ DE URALDE, J. M y RADIRGÁN, M (2013) La economía social en clave internacional. Cuantificación, reconocimiento institucional y visibilidad social en Europa, Iberoamérica y Norte de África, Revesco. Revista de Estudios Cooperativos, $\mathrm{n}^{\circ}$ 112, pp. 122-150

CHEN M. y SNODGRASS D. (2001) Managing resources, activities and risk in urban India: the impact of SEWA Bank. Washington DC, Assesing the impact of microenterprise Services (AIMS), Management Systems International. 
CHESTON S., REED L. y HARPER V. et alii (1999) Comment mesurer la transformation: evaluation et amélioration de l'impact du microcredit, Economie Sociale et Solidaire, Grenoble.

CHU M. Et al. (1996) Corposol/Finansol: Preliminary Analysis of an Institutional Crisis in Microfinance, Global Development Research Center.

COHEN M. (1998) Impact Assessment: The Evolving Agenda, Washington, D.C., Document USAID, Septiembre.

COLLINS D. et al. (2010) Portfolios of the poor: how the world's poor live on $\$ 2$ a day, Princeton University Press.

COPESTAKE J. (2002) Inequality and the polarizing impact of micro-credit: evidence from Zambia’s Copperbelt, Journal of International Development, pp. 743-755.

COPESTAKE J., BHALOTRA S. y JOHNSON S. (2001) Assesing the impact of microcredit: A Zambian case study, Journal of Development Studies, pp. 81-100.

DICHTER T. (2007) The chicken and egg dilema in microfinance: An historical analysis of the sequence of growth and credit in the economic development of the North, Dichter and Harper Edition. Rugby, Warwickshire, UK

DOOCY S., et al. (2005) Credit program outcomes: coping capacity and nutritional status in the food insecure context of Ethiopia, Social Science and Medicine, 60, pp. 2371-2382.

DUPAS P. y ROBINSON J. (2008) Savings constraints and microenterprise development: evidence from a field experiment in Kenya, Los Angeles, UCLA.

DUVENDACK M. et al. (2011) What is the evidence of the impact of microfinance on the well-being of poor people? Social Science Research Unit, Institute of Education, University of London, London.

FECHER, F; CHAVES ÁVILA, R y MONZÓN CAMPOS, J. L (2012) Recent issues in social economy research. Special issue. Annals of Public and Cooperative Economics (vol $83: 3)$

FERNANDO J.L. (2006) Microfinance: Perils and prospects, Routledge, New York.

FISHER G. y GHATAK M. (2011) Spanning the chasm: Uniting the theory and empirics in microfinance research, The Handbook of Microfinance, pp 59-75, World Scientific.

FOURNIER Y. y OUEDRAOGO A. (1996) Les coopératives d'épargne et de crédit en Afrique. Historique et évolution récente, Revue Tiers-Monde, Tome XXXVII, Nº 145.

FRANCESCONI G.N. y HEERINK R. (2010) Ethiopian agricultural cooperatives in an era of global commodity exchange: Does organizational form matter?, Journal of African Economies, 20, pp. 153-177. 
GARCÍA-GUTIÉRREZ, C (2002) Las empresas de participación: características que las definen. Virtualidad y perspectivas en la sociedad de la información. Ciriec España, Revista de Economía Pública, Social y Cooperativa, 40, abril, pp. 99-122.

GEERTZ C. (1961) The Rotating Credit Association: A Middle Rung in Development, Economic and Cultural Change, $\mathrm{N}^{\circ} 1$, pp. 241-263.

GENTIL D. y FOURNIER Y. (1993) Le secteur financier autonome, en Les paysans peuventils devenir banquiers? Epargne et crédit en Afrique, Paris Syros.

GERMIDIS D., KESSLER D. y MEGHIR R. (1991) Systèmes financiers et développement: quel rôle pour les secteurs financiers formels et informels? OCDE, Paris.

GERTRUD R. S. (1994) Groupes financiers informels au Cameroun. En DALE ADAM, DELBERT A.F, Finance informelle dans les pays en développement. Presses Universitaires de Lyon, p. 63.

GETNET K y ANULLO T. (2012) Agricultural cooperatives and rural livelihoods: evidence from Ethiopia, Annals of Public and Cooperative Economics, 83, pp. 181-198.

GHATE et al. (1992) Informal Finance: Somme Findings from Asia, Asian Development Bank.

GINE X. y KARLAN D. (2006) Group versus individual liability: a field experiment in the Philippines, documento de trabajo sobre investigaciones relativas a políticas de desarrollo, Washington DC, Banco Mundial.

GRAHAM D.H. et al. (1987) Rural Finance in Niger: an evaluation and recommendations of reforms, Final Report, Ohio University.

GUBERT F. y ROUBAUD F. (2005) Analyser l'impact d'un projet de microfinance: l'exemple d'Adefi a Madagascar, DIAL (Developpement, Institutions et Analyses de Long Terme), Paris.

HIETALAHTI J. y LINDEN M. (2006) Socioeconomic impacts of microfinance and repayment performance: a case study of the Small Enterprise Foundation, South Africa, Progress in Development Studies, 6 (3), pp. 201-210.

HIMES C. y J. SERVON L. J. (1998) Évaluation de la réussite des clients : évaluation de l'impact d'ACCION sur les microentreprises aux États-Unis, The U.S. Issues Series Document $\mathrm{N}^{\circ}$ 2, abril 1998.

HOLST J.U (1985) Le rôle des institutions financières informelles dans la mobilisation de l'épargne, in Epargne et Développement, Kessler D. et Ullmo P.A. (éd.), Economica, Paris, pp.121-154.

HOSSAIN F. y KNIGHT T. (2008) Financing the poor: can microcredit make a difference? 
Empirical observations from Bangladesh, BWPI Working Paper 38, Manchester, Brooks World Poverty Institute.

HUGON Ph. (1996) Incertitude, précarité et financement local : le cas des économies africaines, Revue Tiers- Monde, $\mathrm{n}^{\circ} 145$, pp. 19-20.

HULME D. Y MOSLEY P. (1996) Finance against Poverty, Vol. I and II London, Routledge.

HULME D. (1997) Impact Assessment Methodologies for microfinance: a Review, Paper prepared for the virtual Meeting of the CGAP Working Group on Impact Assessment Methodologies, AIMS.

HUSAIN Z., MUKHERJEE D. y DUTTA M. (2014) Self-help groups and empowerment of women: Self-selection or actual benefits?, Journal of International Development. Volume 26, Issue 4, pp. 422-437

KARLAN D. y ZINMAN J. (2009) Access to finance, en Handbook of development Economics, vol. 5, Amsterdam, Elsevier.

KARLAN D. y ZINMAN J. (2010) Expanding credit access: Using randomized supply decisions to estimate the impacts, Review of Financial Studies, 23 (1), pp. 433-464.

KARNANI A. (2007) Microfinance misses its marks. Stanford Social Innovation Review. Summer. Disponible en http://www.ssireview.org/articles/entry/microfinance_misses_its_mark

KARNANI A. (2009) Romanticizing the poor. Stanford Social Innovation Review. Winter. Disponible en: http://www.ssireview.org/articles/entry/romanticizing the poor

KENGUE P. (2012) La microfinance en Tunisie et en Egypte: un outil au service du developpement?, Université de Rennes.

KHANDKER S. (1998) Fighting poverty with microcredit: experience in Bangladesh, Nueva York, Oxford University Press.

KHLANDKER S. (2001) Does microfinance really benefit the poor? Evidence from Banglaadesh, Paper delivered at the Asia and Pacific Forum on Poverty: reforming Policies and Institutions for Poverty Reduction, 5-9 February.

KRAHNEM J.P. y SCHMIDT R.H. (1994) Development Finance as Institution Building, West view Press, Boulder (Colorado)

LACALLE CALDERON M., RICO GARRIDO S. y DURAN NAVARRO J. (2008) Estudio piloto de evaluación de impacto del programa de microcréditos de Cruz Roja Española en Ruanda, Revista de Economía Mundial, 19, pp. 83-104.

LEJARRIAGA, G; MARTíN, S y MUÑOZ, A (Dir) (2013). 40 años de historia de las 
Empresas de Participación. Madrid: Editorial Verbum.

LELART M. (1990) La tontine. Pratique informelle d'épargne et de crédit dans les pays en voie de développement, Paris, Editions Aupel-Uref.

LELART M. (2000) L'évolution de la finance informelle au Bénin, Finance informelle et financement du développement, AUPELF/FMA, pp. 17-39, Beyrouth.

LELART M. (1995) Tontines africaines et tontines asiatiques, Afrique Contemporaine, $\mathrm{n}^{\circ} 176$, Octobre-Décembre, 75-86.

LELART M. (1993) Tontines, Innovations et développement, Ponson B. et Schaan J.L. L'esprit d'entreprise, pp. 369-382.

LELART M. (1996) La nouvelle loi sur les mutuelles d'épargne et de crédit dans les pays d'Afrique de l'Ouest : la loi Parmec de l'UEMOA, Monde en développement, vol.24 $\mathrm{n}^{\circ} 94$, pp. 57-69.

LELART M. (2007) Les mutations dans la microfinance : L'expérience du Bénin, Laboratoire d'Economie d'Orléans, Document de recherche $\mathrm{n}^{\circ} 15$.

LELART M. (2002) L'évolution de la finance informelle et ses conséquences sur l'évolution des systèmes financiers, Monde en Développement, T 30-119, pp. 9-20.

LELART M. (1991) Les tontines et le financement de l'entreprise informelle, Notes de Recherche $n^{\circ}$ 91-18, AUPELF-UREF.

LELART M. y GNANSOUNOU S. C. (1994) Tontines et tontiniers sur les marchés africains: le marché Saint-Michel de Cotonou, En ADAMS, D.W. y FITCHETT D.A. (éd): Finance informelle dans les pays en voie de développement, Presses universitaires de Lyon, 1994 115-129.

LIPTON M. (1996) Successes in antipoverty, International Institute of Labor Studies, Geneva.

MARKOWITZ, Harry (1952) Portfolio Selection, Journal of Finance vol VII, nº 1 marzo, pp. $77-91$.

MAYOUX L. (1997) Impact Assessment and Women's Empowerment in Microfinance Programs, Issues for a Participatory Action and Learning Approach, Documento de trabajo presentado en la reunión de GCAP.

MAYOUX L. (1999) Questioning virtuous spirals: microfinance and women's empowerment in Africa, Journal of International Development, 11, pp. 957-984.

MAYOUKOU C. (1994) Le système des tontines en Afrique, un système bancaire informel, Paris, Editions $1^{\text {ee } h a r m a t t a n, ~ C o l l e c t i o n, ~ L o g i q u e s ~ E c o n o m i q u e s, ~ P a r i s . ~}$

MIRACLE M.P. y COHEN L. (1980) Informal Savings Mobilization in Africa, Economic Development and Culturel Change, $\mathrm{N}^{\circ} 24$, pp. 701-724. 
MIX (Microfinance Information Exchange) \& CGAP (Consultative Group to Assist the Poor), MIX microfinance world: Sub-Saharan Africa microfinance analysis and benchmarking report, Washington DC, 2011.

MONTEL P J., AGENOR P R. y HAQUE N. (1993) Informal Financial Markets in Developing Countries, Blackwell, Oxford.

MORDUCH J. (1998) Does Microfinance really help the poor? New evidence on flagship programs in Bangladesh, World Bank, Washington.

MOSLEY P. y HULME D. (1998) Microenterprise finance: is there a conflict between growth and poverty alleviation?, World Development, 26 (5), pp. 783-790.

NARRILLOS, H. (2010) El SROI (Social Return on Investment): un método para medir el impacto social de las inversiones. Análisis Financiero n ${ }^{\circ} 113$, pp. 34-43.

NARRILlOS, H. (2013) Economía Social. Valoración y medición de la inversión social (método SROI). Ecobook. Madrid.

NANOR M.A. (2008) Microfinance and its impact on selected districts in Eastern region of Ghana, College of Art and Social Sciences, Kwame Nkrumah University of Science and NOVAK M. (1990) On ne prête qu'aux riches, Paris, L“eharmattan.

ODELL K. (2010) Measuring the impact of microfinance: taking another look, Grameen Foundation, Washington DC.

PARRAMON, E (2014) Claves para entender la banca en la sombra: Shadow Banking. Análisis Financiero 125, pp. 69-78

PATTEN R., ROSENGARD J. y JOHNSON D. Jr. (2001) Microfinance Success Amidst Macroeconomic Failure: The Experience of Bank Rakyat Indonesia During the East Asian Crisis. World Development. 29 (6), pp. 1057-1069.

PRONYK P.M. et al. (2008) A combined microfinance and training intervention can reduce HIV risk behavior in young female participants, AIDS, 22, pp. 1659-1665.

RAHMAN A. (1998) A micro-credit initiative for equitable and sustainable development: who pays?, World Development, 26 (1), pp. 67-82.

ROBINSON M.S. (2001) The microfinance revolution: sustainable finance for the poor, Washington, DC, The World Bank.

ROY A. (2010) Poverty capital: Microfinance and the making of development, Routledge, New York.

RUTHERFORD S. (1996) A critical typology of financial services for the poor, Action Aid \& Oxfam, London.

SCHULER S.R., HASHEMI S.M. y RILEY A.P. (1997) The influence of women's changing 
roles and status in Bangladesh 's fertility transition: evidence from a study of credit programs and contraceptive use. World Development, 25 (4), pp. 563-576.

SEIBEL H. (2005) The microbanking division of Bank Rakyat Indonesia: a flagship of rural microfinance in Asia, ITDG Publications, Rugby.

SENIGAGLIA, C (2008) Europa y las opciones éticas de la cooperación al desarrollo. Revista Universitaria Europea 8, pp. 105-120.

SERVET J. M. (1995) Formes, raisons et devenir des modes informels d'épargne. En SERVET, J. M. (ed): Epargne et liens sociaux : Etude comparées d'informalités financière, Association d'Economie Financière, París, pp. 26-29.

SERVET J.M. (2000) Exclusion et liens financiers. Rapport du Centre Walras 1999-2000, Economica.

SERY A. (2012) Le micro-crédit: l'empowerment des femmes ivoiriennes Tesis doctoral, Doctorat de 1' Université Paul-Valéry, Montpellier.

SHIMAMURA Y. y LASTARRIA-CORNHIEL S. (2009) Credit program participation and child schooling in rural Malawi, Worl Development, 38, pp. 567-580.

SHREINER M. (1997) A Framework for the Analysis of the Performance and Sustainability of Subsidized Microfinance Organization with Application to Bancosol of Bolivia and the Grameen Bank of Bangladesh, Dissertion Grad Sch of the Ohio State Univ.

SPARREBOOM P. y DUFLOS E. (2012) Financial inclusion in China, CGAP report.

SSEWAMALA F.M. et al. (2010) Gender and the effects of an economic empowerment program on attitudes toward sexual risk-taking among AIDS orphaned adolescent youth in Uganda, Journal of Adolescent Health, 46 (4), pp. 372-378.

TOWNSEND R. y KABOSKI J. (2009) The impact of credit on village economies, Departamento de economía del MIT, documento de trabajo ${ }^{\circ}$ 09.13. Mass: MIT.

UDRY C. y ARYEETEY E. (2010) Creating Property rights: Land Banks in Ghana, American Economic Review Papers and Proceedings. 100 (2), pp. 130-134.

UNICEF (1997) Give us credit, Division of Evaluation, Policy and Panning, Geneva.

UTUN W. (1957) Interest Rates Outside the Organized Money of Underdeveloped Coutries, FMI, Staff Papers, Vol.VI, pp. 80-142.

VAN ROOYEN C., STEWART R. y DE WET T. (2012) The impact of microfinance in SubSaharan Africa: a systematic review of evidence, World Development, 40, pp. 2249-2262.

VARGAS, A y LEJARRIAGA, G. (Coord) (2002). Las empresas de participación en Europa: El reto del siglo XXI. Escuela de Estudios Cooperativos. Universidad Complutense de Madrid. Madrid. 
VONDERLACK R.M. y SCHREINER M. (2003) Women, Microfinance, and Savings: Lessons and Proposals, Working paper, Center for Social Development, Washington, St. Louis University.

WANYAMA F. et al. (2008) Encountering the evidence: cooperatives and poverty reduction in Africa, Working Papers on Social and Cooperative Entrepreneurship, Maseno University, Kenya.

WEBER H. (2006) The global political economy of microfinance and poverty reduction: Locating local livelihoods in political analysis, Routledge, London.

WOLLER G. et al. (1998) Where to Microfinance?, International Journal of Economic Development, 1, pp. 29-64.

WRIGHT G.A.N. (2000) Microfinance systems: designing quality financial services for the poor, Zed Books, London.

YARON J. (1992) Successful Rural Financial Institutions. Washington. World Bank Discussion Paper $N^{\circ} 150$.

YARON J. (1994) What makes rural finance institutions successful?, Washington, Research Observe, World Bank.

YUNUS M. (1994) Grameen Bank, as I see it, Grameen Bank.

YUNUS M. (1999) Banker to the poor: micro lending and the battle against world poverty, Public Affairs, New York.

YUNUS M. (2006) ¿Es posible acabar con la pobreza?, Editorial Complutense. Madrid

ZAMAN H. (2001) Assessing the poverty and vulnerability impact of micro-credit in Bangladesh: a case of BRAC, World Development Report 2000/2001, World Bank, Washington. 